\title{
Urodynamic and neurophysiological evaluation in Parkinson's disease and multiple system atrophy
}

\author{
F Stocchi, A Carbone, M Inghilleri, A Monge, S Ruggieri, A Berardelli, M Manfredi
}

\begin{abstract}
Aims-To determine whether Parkinson's disease and multiple system atrophy each has a distinct pattern of micturition abnormalities and whether a urodynamic evaluation could be useful in the differential diagnosis between the two diseases. Methods-Sixty two patients (30 with Parkinson's disease and 32 with multiple system atrophy) underwent a complete urodynamic evaluation and neurophysio-
\end{abstract} logical testing.

Results-Of the parkinsonian patients $36.6 \%$ had normal micturition findings with normal bladder sensitivity; $26 \cdot 7 \%$ had delayed or incomplete pelvic floor relaxation; $26.7 \%$ had hyperreflexia with vesicosphincteric synergy; and $10 \%$ had hyperreflexia with vesicosphincteric synergy associated with incomplete pelvic floor relaxation. Parkinsonian patients with a normal urodynamic pattern had significantly less severe disease and a shorter duration of disease in years than those who had abnormal patterns. Patients with hyperreflexia had significantly higher severity of disease.

Department of Neurological Sciences, University of Rome,

"La Sapienza", Rome, Italy

F Stocchi

$M$ Inghilleri

A Monge

$S$ Ruggieri

A Berardelli

$M$ Manfredi

Department of

Urology, University of

Rome, "La Sapienza", Rome, Italy

A Carbone

Institute of

Neurosciences

"Neuromed", Pozzilli

(IS), Italy

$S$ Ruggieri

M Manfredi

Institute of Neurology

"A Benedetti",

Vicenza, Italy

F Stocchi

A Monge

Correspondence to:

Dr Fabrizio Stocchi, Dip di

Scienze Neurologiche, Vial

dell' Università, 30,00185

Roma, Italy.

Received 27 March 1996

and in final revised form

13 November 1996

Accepted 10 December 1996
All the patients with multiple system atrophy had hyperreflexia with synergy. Two urodynamic patterns were identified: hyperreflexia with vesicosphincteric synergy $(90.6 \%$ of patients), and hyperreflexia with vesicosphincteric synergy and incomplete pelvic floor relaxation (in $\mathbf{9 \cdot 4 \%}$. Hyperreflexia with synergy correlated neither with the severity nor with the duration of disease. Sphincter EMG analysis showed that all the parkinsonian patients had normal sphincter EMG whereas 24 of the 32 patients with multiple system atrophy had neurogenic signs. Conclusions-Urodynamic evaluation and sphincter EMG are both useful tests in the differential diagnosis between Parkinson's disease and multiple system atrophy. Urodynamic findings may be abnormal before patients with multiple system atrophy reach an advanced stage of the disease. Recordings of EMGs from perineal muscles become abnormal as the disease progresses in multiple system atrophy but not in Parkinson's disease.

(F Neurol Neurosurg Psychiatry 1997;62:507-511)
Keywords: urodynamic; sphincter electromyography; Parkinson's disease; multiple system atrophy

The term multiple system atrophy encompasses several neurodegenerative syndromes (striatonigral degeneration, olivopontocerebellar atrophy, and Shy-Drager disease), all characterised by coexisting extrapyramidal, cerebellar, pyramidal, and autonomic involvement. ${ }^{1-3}$

During the course of their disease patients with multiple system atrophy commonly have urinary dysfunction consisting of urinary frequency, urinary urgency, and urge incontinence or nocturia, or both abnormalities. ${ }^{4-6}$ Similar urinary dysfunctions can also be present in patients with Parkinson's disease, ${ }^{78}$ but the incidence of urinary symptoms and the correlation with the severity of the disease is not well defined.

In many cases it is clinically difficult to differentiate Parkinson's disease from multiple system atrophy. Although the sphincter EMG may be abnormal in multiple system atrophy but not in Parkinson's disease, it is not a reliable test. ${ }^{6} 9^{10}$

The aim of this study was to define the specific urodynamic and sphincter EMG patterns in Parkinson's disease and multiple system atrophy. We also compared the findings to determine whether the two diagnostic tests would be useful in differentiating these two disorders.

\section{Methods}

PATIENTS

Sixty two patients ( 25 women and 37 men) agreed to participate in this study after full disclosure of its purpose, risks, and potential benefits. Thirty patients (12 women and 18 men; mean age $60 \cdot 8$ (SD 8.3), range $50-75$ years) had idiopathic Parkinson's disease diagnosed by clinical examination, CT, MRI, and pharmacological tests. All patients responded well to an acute challenge with either levodopa or apomorphine and had a sustained response to chronic treatment with levodopa. The mean duration of disease was 10.3 (SD 6.5) years, the mean duration of levodopa therapy was 9.6 (SD 6.3) years, and at the time of the study their daily levodopa requirement was 723 (SD 345) $\mathrm{mg}$. The mean severity of disease was 3.4 (SD 0.9) (Hoehn and Yahr scale). All the parkinsonian patients were 
Clinical features of 32 patients with multiple system atrophy

\begin{tabular}{ll}
\hline Sex: & $19(59 \cdot 4 \%)$ \\
M & $13(40 \cdot 6 \%)$ \\
F & \\
Age: & $43-79$ \\
Range & 63 \\
Mean & $19(59 \cdot 4 \%)$ \\
Pyramidal signs & $10(31 \cdot 2 \%)$ \\
Cerebellar signs & $30(93 \cdot 7 \%)$ \\
Parkinsonism & $16(50 \cdot 0 \%)$ \\
Tremor & $17(53 \cdot 1 \%)$ \\
Postural faintness & $15(46 \cdot 9 \%)$ \\
Laryngeal stridor & $9(28 \cdot 1 \%)$ \\
Dysartrophonia & $13(40 \cdot 6 \%)$ \\
Response to levodopa: & $12(37 \cdot 5 \%)$ \\
None & $7(21 \cdot 9 \%)$ \\
Moderate & Pronounced \\
\hline
\end{tabular}

examined during the "off" (unmedicated) phase and the "on" (optimally medicated) phase obtained with subcutaneous injection of apomorphine (4-6 mg).

The study group with possible multiple system atrophy consisted of 32 patients (13 women and 19 men; mean age 63.3 (SD 8.1) range 43-79 years). Their duration of disease was $7 \cdot 1$ (SD $4 \cdot 6)$ and 19 out of 32 were on levodopa therapy (mean daily dosage 538 (SD 244) mg). Multiple system atrophy was diagnosed according to the presence of at least two of the following clinical signs: parkinsonian, cerebellar, autonomic, or pyramidal signs (evidence of pyramidal signs was the presence of extensor plantar response or the combination of hyperreflexia and equivocal plantar responses) ${ }^{11}$; acute pharmacological tests (apomorphine (subcutaneous injection of $4 \mathrm{mg}$ ) and levodopa (250 mg plus $50 \mathrm{mg}$ PDI)); and subacute pharmacological tests ( 30 days of oral levodopa treatment). A poor response to apomorphine or levodopa was defined as a clinical benefit of less than $30 \%$ evaluated by the unified Parkinson's disease rating scale (UPDRS). Duration of disease was $7 \cdot 1$ (SD $4 \cdot 6$ ) years and the duration of therapy was $6 \cdot 2$ (SD 5.7) years. Nineteen patients had pyramidal signs; 10 had cerebellar signs; and 17 had hypotension. All patients with multiple system atrophy underwent CT or MRI studies. In 11 patients magnetic resonance imaging showed cerebellar atrophy or brainstem atrophy (table). Severe symptomatic orthostatic hypotension and faecal incontinence could have interfered with the execution of the tests and also with the blind study design; therefore, patients with these symptoms were excluded from the study. Patients with a mini mental state score below 28 or who had had previous pelvic surgery were also excluded. In all subjects anticholinergic drugs were withdrawn one week before the study to exclude possible drug induced vesical hypocontractility or incomplete relaxation of the pelvic floor. Other dopaminergic drugs were stopped the night before the study.

\section{URODYNAMIC EVALUATION}

The study (cystometry, flowmetry, micturitional study, EMG, and urethral pressure profile) was done by a urologist blind to diagnosis. Wiest 6000 and 6000-plus urodynamic devices were used. For cystometry, the bladder was filled at an average infusion rate of 40 $\mathrm{ml} / \mathrm{min}$ and bladder pressure was measured with a two way Nelaton catheter. Patients were seated with the trunk bent backwards by $45^{\circ}$ and the legs raised in the gynaecological position throughout the study. During cystometry and the micturitional study the muscular activity of the perineal floor during bladder filling and micturition was assessed with perineal EMG by means of a concentric needle electrode (Vickers MCD 25). The normal bladder can fill to $400-500 \mathrm{ml}$ with only a slight increase in pressure (less than $15 \mathrm{~cm}$ $\mathrm{H}_{2} \mathrm{O}$ ), peak filling ultimately producing a moderately strong desire to void. In a bladder with normal sensation, normal high compliance and no involuntary contractions during the filling phase the bladder content increases in volume, provoking no sensation until about $150 \mathrm{ml}$ has been infused.

\section{NEUROPHYSIOLOGICAL EVALUATION}

Sphincter EMGs were recorded by a neurologist blinded to diagnosis, with a concentric needle electrode (Vickers MDC 25) inserted into the bulbocavernosus muscle and external anal sphincter muscle (bandwidth $20-10 \mathrm{kHz}$ or $100-10 \mathrm{kHz})$. The EMG activity was recorded at rest and during slight and maximal voluntary activation of the muscles studied. The bulbocavernosus muscle reflex was evoked by stimulating the dorsogenital nerve with surface electrodes placed on the clitoris or penis and responses were recorded from the bulbocavernosus muscle using a concentric needle electrode. Motor unit action potentials were considered abnormal if they lasted longer than $10 \mathrm{~ms}$ and if their amplitude exceeded 1 $\mathrm{mV}$ or if more than $27 \%$ were polyphasic (upper limit for our normal age matched population). The bulbocavernosus muscle reflex was considered abnormal if it was absent or when the latency of the oligosynaptic response exceeded the normal value $(30.2$ (SD 3.9$) \mathrm{ms})$ obtained in 10 age matched normal subjects.

All data are expressed as means (SD). Statistical significance of the data was assessed with the $t$ test for unpaired data. $\mathbf{P}$ Values $\leqslant 0.05$ were considered to indicate significance.

\section{Results}

PATIENTS WITH PARKINSON'S DISEASE

Parkinsonian patients were classified into four groups according to their urodynamic results:

(1) Eleven patients $(36.6 \%)$ had normal urodynamic findings. These patients had normal bladder sensitivity. They reported the first desire to void when bladder filling reached 90 to $160 \mathrm{ml}$. The maximum bladder capacity was $410 / 530 \mathrm{ml}$. None of these patients had involuntary contractions during the study. They all had normal detrusor contraction and perineal activity during micturition.

(2) Eight patients $(26.7 \%)$ had normal bladder sensitivity and normal cystometric findings but showed delayed or incomplete relaxation of the perineal floor. On being asked to initiate micturition they were unable to relax the perineal muscle immediately and completely. 
(3) Eight patients $(26 \cdot 7 \%)$ had a sudden, involuntary detrusor contraction that occurred during bladder filling when the filling volume reached 80 to $220 \mathrm{ml}$. The contraction was uncontrolled and was accompanied by various degrees of incontinence. We defined this urodynamic pattern as hyperreflexia with vesicosphincteric synergy.

(4) In three patients (10\%) detrusor hyperreflexia with vesicosphincteric synergy was associated with incomplete relaxation of perineal muscle.

Two of the 30 patients with Parkinson's disease (group 3) complained of frequency and urgency of micturition at the time of the study and only one had urinary incontinence during the day or at night (group 4).

In the patients who had delayed or incomplete perineal floor relaxation (groups 2 and 4) subcutaneous injection of apomorphine (4 mg) greatly improved perineal muscle control. In none of the patients who had hyperreflexia with vesicosphincter synergy did the drug improve this symptom (group 3). In the patients who had normal urodynamic findings (group 1), apomorphine injection left these unchanged.

The patients who had hyperreflexia with synergy (group 3) had more severe disease and longer duration of disease than the remaining 19 patients (groups 1 and 2) (Hoehn and Yahr stage 4.1 (SD 0.54) and disease duration 14.5 (SD 7.49) years in the hyperreflexia-synergy group versus Hoehn and Yahr stage 3.06 (SD 1.0 ) and disease duration $7 \cdot 82$ (SD 4.71) years in the other patients; $P<0.01$, unpaired $t$ test).

Among the patients with normal urodynamic evaluation (groups 1 and 2) the eight patients with delayed or incomplete perineal floor relaxation (group 2) had more severe disease and longer duration of disease than the 11 patients (group 1) without delayed perineal floor relaxation (Hoehn and Yahr stage 3.6 (SD 0.5) and disease duration 10.3 (SD 5.2) years versus Hoehn and Yahr stage 2.5 (SD $1 \cdot 1)$ and disease duration $5.5(\mathrm{SD} 2 \cdot 7)$ years; $\mathrm{P}<0.01$, unpaired $t$ test).

All the parkinsonian patients had normal sphincter EMG findings and bulbocavernosus muscle reflex (31.8 (SD 2.9) $\mathrm{ms}$ ).

\section{MULTIPLE SYSTEM ATROPHY}

All the patients with multiple system atrophy had hyperreflexia with vesicosphincteric synergy. This involuntary detrusor contraction was uncontrolled and in 29 of the 32 patients $(90.6 \%)$ it was accompanied by various degrees of incontinence (group 1). In the remaining three patients $(9 \cdot 4 \%)$ hyperreflexia with vesicosphincteric synergy was associated with incomplete or delayed pelvic floor relaxation (group 2).

None of the 32 patients with multiple system atrophy had residual urinary volume.

Sphincter EMG showed that 24 of the 32 patients $(74 \%)$ had denervation and chronic neurogenic signs. The EMG abnormalities showed the same degree of severity in the two muscles examined (bulbocavernosus and external anal sphincter). All patients had a normal bulbocavernosus muscle reflex (30.4 (SD 30 ) $\mathrm{ms}$ ). The 24 patients with neurogenic abnormalities had a significantly longer duration of disease than the other eight patients $(5.7$ (SD 2.8) $\vee 2.1$ (SD 0.9) years, $P<0.001)$. The presence of neurogenic signs did not correlate with the patients' age.

Thirteen of the 32 patients complained of frequency and urgency of micturition at the time of study. Although all had abnormal urodynamic examination only 10 of these 13 showed abnormal EMG findings. Five additional patients also had urinary incontinence during the day or at night. This urinary leakage was preceded by a feeling of urgency, but the patients could not prevent urine loss or interrupt the stream once it started. All five patients had abnormal urodynamic and EMG examination. The remaining 14 patients had no urinary symptoms. All of them had urodynamic abnormalities but only nine had neurogenic signs at the EMG.

The eight patients who had normal sphincter EMGs at the first examination underwent new sphincter EMG every six months. Three of these patients complained of frequency and urgency of micturation at the time of the study and all of them had hyperreflexia with vesicosphincter synergy. In all these patients neurogenic signs eventually developed within six to 24 months. Their bulbocavernosus muscle reflex remained normal.

\section{Discussion}

All the patients with multiple system atrophy but only $26.7 \%$ of those with Parkinson's disease had hyperreflexia with vesicosphincter synergy. In $36.6 \%$ of the Parkinsonian patients urodynamic studies gave normal findings and in $36.6 \%$ there was an isolated incomplete relaxation of the pelvic floor. The incidence of hyperreflexia-synergy with incomplete relaxation was similar in the two groups (about $10 \%)$.

In this study the percentage of abnormalities present in the patients with Parkinson's disease was lower than that reported in other studies. ${ }^{1213}$ These discrepant findings may reflect clinical differences in the patients studied; in one study, eight of the 29 patients had had prostatic surgery. ${ }^{13}$

Urodynamic evaluation in parkinsonian patients showed that hyperreflexia with vesicosphincteric synergy was a more frequent finding in patients who had more severe motor disorders and longer duration of disease. In multiple system atrophy, hyperreflexia was already present even in patients with less advanced disease.

Neurophysiological evaluation also showed differences between Parkinson's disease and multiple system atrophy. EMG recordings from the external anal sphincter and bulbocavernosus muscle yielded normal findings in all the parkinsonian patients whereas in 24 of our 32 patients with multiple system atrophy they disclosed neurogenic abnormalities. These results agree with those of Pramstaller et al, ${ }^{10}$ 
who reported abnormal sphincter EMG findings in about $90 \%$ of their patients. This suggests that multiple system atrophy causes neuronal degeneration not only of Onuf's nucleus, which innervates the external anal and striated urethral sphincters, but also of the pudendal motor neurons innervating the bulbocavernosus muscle. The normal bulbocavernosus muscle reflex excludes disorders affecting the afferent pathway of the reflex. In the group of eight patients who had less severe disease and a shorter duration of disease than the other 24 patients, sphincter EMG obtained at the time of urodynamic evaluation showed normal findings. Interestingly, in all of these eight patients neurogenic signs developed within eight to 24 months. The appearance of neurogenic bladder dysfunction later in the course of the disease agrees with published data indicating that although the sphincter EMG examination is a poorly sensitive test it is highly disease specific. ${ }^{6910}$

Abnormalities of sphincter EMG activity similar to those present in multiple system atrophy may also be present in patients with other neurological diseases with parkinsonism, such as progressive supranuclear palsy. ${ }^{14}$ This suggests that sphincter EMG is an index of Onuf's nuclei degeneration. In other conditions with anterior horn loss, such as amyotrophic lateral sclerosis, the anal sphincter EMG examination is normal. ${ }^{15}$

The coordinating centre of micturition is thought to be in the dorsal tegmentum of the pons. Neural pathways from this centre traverse the spinal cord to reach the sacral spinal cord. Inhibitory control from medial frontal lobes is exerted on the pontine micturition centre. Micturition is also influenced by the anterior cingulate gyrus, the locus coeruleus, the pontomesencephalic grey matter, and the nucleus tegmentolateralis dorsalis. The finding of hyperreflexia with vesicosphincteric synergy suggests a suprapontine lesion. Among the other brain structures that regulate micturition, the globus pallidus has been reported to suppress spontaneous detrusor contractions $^{1617}$ and the subthalamus and substantia nigra to inhibit reflex bladder contractions. Patients with multiple system atrophy have evidence of neuronal degeneration of the striatum (mainly putamen), substantia nigra, locus coeruleus, cerebellar cortex, pontine nuclei, inferior olives, the intermediolateral cell column of the spinal cord, and Onuf's nucleus in the spinal cord. ${ }^{18}{ }^{19}$ In multiple system atrophy damage to brain structures that inhibit bladder contractions may therefore be the cause of the hyperreflexia.

A second possibility is that the urodynamic abnormalities present in patients with multiple system atrophy arise from involvement of the corticospinal tracts. Ample evidence shows degeneration of these tracts. Pyramidal signs have been reported in $62.5 \%$ of these patients. ${ }^{11}$ Detrusor hyperreflexia is present in patients with multiple sclerosis with lower limb pyramidal involvement and has been attributed to interruption of pathways between the pons and the sacral cord. ${ }^{20}$ Although we cannot completely exclude this possibility in our series, the fact that we also found urodynamic abnormalities in the patients without clinical evidence of pyramidal signs makes it unlikely. Urinary abnormalities in Parkinson's disease may be due to a lack of inhibition from the substantia nigra on the detrusor activity. ${ }^{16} 17$ The delayed or incomplete voluntary relaxation of the pelvic floor (sphincteric bradykinesia) found in our patients with Parkinson's disease may be due to physiological mechanisms similar to those proposed for the bradykinesia ${ }^{21}$; hence the reversal of this symptom by apomorphine.

In multiple system atrophy, urodynamic studies can be a useful diagnostic test especially in the early stage of disease. Indeed, the parkinsonian patients with normal urodynamic findings (groups 1 and 2) had similar durations of disease to the patients with multiple system atrophy who showed abnormal findings (7.8 $v 7 \cdot 1$ years) and the parkinsonian patients with abnormal urodynamic findings (group 3) had much longer duration of disease (14.5 years). On the other hand, sphincter EMG may be normal during the early stage of the disease when urodynamic evaluation is already abnormal and the patients may have urinary symptoms. On clinical features alone multiple system atrophy is difficult to distinguish from Parkinson's disease. ${ }^{2322}$ At the early stage both diseases give rise to parkinsonian signs. To complicate matters, some patients, at least in the early stage of their disease, respond well to levodopa therapy. Because symptoms and signs reflecting damage to other systems, present only in patients with multiple system atrophy, often arise later they do not help the early differential diagnosis. Although imaging abnormalities have been shown within the basal ganglia and the pontocerebellar system single criteria do not suffice for the diagnosis. ${ }^{23}$ Even F-dopa PET is not completely reliable in distinguishing Parkinson's disease from multiple system atrophy. ${ }^{24}$

Our findings suggest that sphincter EMG and urodynamic evaluation could be useful in distinguishing Parkinson's disease from multiple system atrophy. The techniques have limitations. For example, urodynamic and neurophysiological abnormalities similar to those in our patients with neurodegenerative disorders commonly occur in men with prostatic hypertrophy and bladder neck stenosis and women with genuine stress incontinence due to urethral prolapse.

1 Graham JG, Oppenheimer DR. Orthostatic hypotension and nicotine sensitivity in a case of multiple system atrophy. $\mathcal{F}$ Neurol Neurosurg Psychiatry 1969;32:28-34.

2 Quinn N. Multiple system atrophy-the nature of the beast. F Neurol Neurosurg Psychiatry 1989;special suppl: $78-89$.

3 Wenning GK, Ben Shlomo Y, Magalhaes M, Daniel SE, Quinn NP. Clinical features and natural history of multiple system atrophy: an analysis of 100 cases. Brain ple system atroph

4 Murnagha GF. Neurogenic disorders of the bladder in parkinsonism. Br f Urol 1961;333:403-9.

5 Beck O, Beths CD, Fowler CS. Genitourinary dysfunction in multiple system atrophy: clinical features and treatment in 62 cases. $\mathcal{F}$ Urol 1994;151:1336-41.

6 Kirby R, Fowler C, Gosling J, Bannister R. Urethro-vescical dysfunction in progressive autonomic failure with multi- 
ple system atrophy. $\mathcal{F}$ Neurol Neurosurg Psychiatry 1986; 49:554-62.

7 Andersen JT, Hebjorn S, Frimodt-Moller C, Walter S, Worm-Petersen J. Disturbances of micturition in Parkinson's disease. Acta Neurol Siand 1976;53:161-70.

8 Pavlakis AJ, Siroky MB, Goldstein IL, Krane RJ. Urologic findings in Parkinson's disease. F Urol 1983;129:80-3.

9 Eardley I, Quinn NP, Fowler CJ, Kirby RS, Parkhouse HF, Marsden CD, Bannister R. The value of urethral sphincter electromyography in the differential diagnosis of parkinsonism. Br f Urol 1989;64:360-2.

10 Pramstaller PP, Wenning GK, Smith SJM, Beck RO Quinn NP, Fowler CS. Nerve conduction studies, skeletal muscle EMG, and sphincter EMG in multiple system atrophy. F Neurol Neurosurg Psychiatry 1995;58:618-21.

11 Quinn NP, Marsden CD. The motor disorder of multiple system atrophy. 7 Neurol Neurosurg Psychiatry 1993;56: system at.

12 Andersen JT, Bradley WE. Cystometric, sphincter and electromyographic abnormalities in Parkinson's disease. electromyographic abnormalities in Parkinson's disease.

13 Berger Y, Salinas JN, Blaivas JG. Urodynamic differentiation of Parkinson's disease and Shy-Drager syndrome. Neurourology and Urodynamics 1990;9:117-21.

14 Vallderiola F, Valls-Solè J, Tolosa ES, Marti MJ. Striated anal sphincter denervation in patients with progressive supranuclear palsy. Mov Disord 1995;10:550-5.

15 Sakuta $M$, Nakanishi T, Toyokura Y. Anal muscle electromyograms differ in amyotrophic lateral sclerosis and Shy-Drager syndrome. Neurology 1978;28:1289-93.
16 Lewin RJ, Dillard GV, Porter RW. Extrapyramidal inhibition of the urinary bladder. Brain Res 1967;4:301-7.

17 Raz S. Parkinsonism and neurogenic bladder-experimental and clinical observation. Urol Res 1976;4:133-5.

18 Fearnley JM, Lees AJ. Striatonigral degeneration: a clinico pathological study. Brain 1990;113:1823-42.

19 Bannister R, Oppenheimer DR. Degenerative disease of the nervous system associated with autonomic failure. Brain 1972;95:457-74.

20 Betts CD, O'Mellow MT, Fowler CJ. Urinary symptoms and the neurological features of bladder dysfunction in multiple sclerosis. F Neurol Neurosurg Psychiatry 1993; 56:245-50.

21 Agostino R, Berardelli A, Formica A, Accornero N, Manfredi M. Sequential arm movements in patients with Parkinson's disease, Huntington disease and dystonia. Brain 1992;115:1481-95.

22 Albanese A, Colosimo C, Bentivoglio AR, Fenici R, Melillo G, Colosimo C, Tonali P. Multiple system atrophy presenting as parkinsonism: clinical features and diagnostic senting as parkinsonism: clinical features and diagnostic

23 Schulz JB, Klockgether T, Peterson D, et al. Multiple system atrophy: natural history, MRI morphology, and tem atrophy: natural history, dopamine receptor imaging with IBZM-SPECT. $\mathcal{f}$ Neurol Neurosurg Psychiatry 1994;57:1047-56.

24 Burn DJ, Sawle GU, Brooks DJ. Differential diagnosis of Parkinson's disease, multiple system atrophy and SteelRichardson-Olszewski syndrome: discriminant analysis of striatal 18-F-dopa PET data. $f$ Neurol Neurosurg Psychiatry 1994;57:278-84. 